\title{
Avaliação e Enfrentamento de Problema de Crianças e Adolescentes com Diferentes Níveis de Comportamentos Externalizantes
}

\author{
Daniela Sacramento Zanini \\ Pontificia Universidade Católica de Goiás \\ Goiânia, GO, Brasil \\ Juliane Callegaro Borsa \\ Pontificia Universidade Católica do Rio de Janeiro \\ Rio de Janeiro, RJ, Brasil
}

\section{RESUMO}

Pesquisas evidenciam uma relação entre comportamentos externalizantes, percepção e enfrentamento de problemas. Este estudo tem como objetivo comparar a avaliação e enfrentamento de crianças e adolescentes com baixas, médias e altas pontuações em comportamento externalizantes. Participaram 512 crianças e adolescentes de 11 a 18 anos incompletos que responderam ao Coping Response Inventory-Y (CRI-Y) e Youth Self Report (YSR). Não foram observadas diferenças estatisticamente significativas nos comportamentos agressivos ou de quebra de regras por sexo. Contudo, observou-se diferenças estatisticamente significativas entre a avaliação e enfrentamento segundo os níveis de comportamentos agressivos ou de quebrar regras. Em conjunto, os dados apresentados neste estudo parecem corroborar a literatura que aponta a existência de uma distorção perceptiva e baixas habilidades de coping entre crianças e adolescentes com problemas de externalizantes. Tais informações podem ser especialmente úteis para o desenvolvimento de programas de manejo de estresse, correção cognitiva e treinamento em habilidades de enfrentamento.

Palavras-chave: Enfrentamento; Distúrbios do comportamento; Comportamento agressivo.

\section{ABSTRACT}

\section{Appraisal and Coping of Children and Youths with Different Levels of Externalizing Behavior}

Studies show a relationship between externalizing behavior, perception and coping. This study aims to compare the appraisal and coping of children and adolescents with low, medium and high scores on externalizing behavior. Participants were 512 children and adolescents aged 11 to incomplete 18, who responded to Coping Response Inventory- Y (CRI- Y) and Youth Self Report (YSR). No statistically significant differences were found on aggressive behavior or breaking rules by sex. However, there was a statistically significant difference between appraisal and coping according to the levels of aggressive behavior or breaking rules. Taken together, the data presented in this study seem to corroborate the literature that points to the existence of a perceptual distortion and low coping skills among children and adolescents with externalizing problems. This information can be especially useful for the development of stress management, cognitive correction and training in coping skills programs.

Keyword: Coping; Behavior disorders; Aggressive behavior.

\section{RESUMEN}

\section{Evaluación y Afrontamiento de Niños y Adolescentes con Distintos Niveles de Conductas Externalizantes}

Los estudios muestran una relación entre las conductas externalizantes, la percepción y el afrontamiento de problemas. Este estudio tiene como objetivo comparar la evaluación y afrontamiento de los niños y adolescentes con puntuaciones bajas, medias y altas en comportamientos externalizantes. Participaron 512 niños y adolescentes de 11 a 18 años incompletos que respondieron al Coping Response Inventory-Y (CRI- Y) y Youth Self Report (YSR). No fueron observadas diferencias estadísticamente significativas en el comportamiento agresivo o romper reglas por sexo. Sin embargo, hubo una diferencia estadísticamente significativa entre la evaluación y afrontamiento de acuerdo con los niveles de comportamiento agresivo o romper reglas. En conjunto, los datos presentados en este estudio parecen corroborar la literatura que apunta la existencia de una distorsión en la percepción y bajas habilidades de afrontamiento de los niños y adolescentes con problemas externalizantes. Esta información puede ser especialmente útil para el desarrollo de programas de combate al estrés, la corrección cognitiva y el entrenamiento en habilidades de afrontamiento.

Palabras clave: Afrontamiento; Problemas de la Conducta; Conducta agresiva. 
Os problemas de comportamento na infância e na adolescência são definidos por condutas socialmente inadequadas que prejudicam o relacionamento interpessoal, seja com os pares seja com os adultos (Bolsoni-Silva \& Del Prette, 2003). Estudos apontam para a alta prevalência de problemas de comportamento em crianças e adolescentes (Anselmi et al., 2010; Fleitlich-Bilyk \& Goodman, 2004) e para o impacto negativo que estes comportamentos podem ter ao longo do desenvolvimento (Homem, Gaspar, Azevedo, \& Canavarro, 2013).

A literatura apresenta diferentes propostas para compreender e classificar os problemas de comportamentos. Thomas Achenbach, desenvolveu o Sistema Achenbach de Avaliação Baseada em Evidências (ASEBA), o qual propõe que os problemas de comportamento podem ser classificados como internalizantes ou externalizantes (Achenbach \& Rescorla, 2010). Os problemas internalizantes, mais comuns em meninas, podem ser observados quando há excesso de preocupação, isolamento social, tristeza, timidez, insegurança e medos. Já os problemas externalizantes, mais comuns em meninos, são caracterizados pela impulsividade, agressões físicas ou verbais, agitação, provocações e comportamentos opositores (Emerich, Rocha, Silvares \& Gonçalves, 2012).

A partir da perspectiva empiricamente baseada de classificação, Achenbach desenvolveu uma série de instrumentos para avaliação de problemas de comportamento em indivíduos de diferentes faixas etárias e destinados a diferentes tipos de informantes. O mais conhecido é o Child Behavior Checklist (CBCL 6/18), desenvolvido para avaliar a competência social e os problemas emocionais e de comportamento de crianças e adolescentes entre 6 e 18 anos a partir da resposta de pais, mães e outros cuidadores. Também amplamente utilizado, o Youth Self Report (YSR) é a versão de autorrelato destinada a avaliar os problemas de comportamento e de competência social de adolescentes entre 11 e 18 anos (Bordin et al., 2013).

Os problemas de comportamento externalizantes compõem algumas das principais características dos comportamentos antissociais (APA, 2003) e por esse motivo, muitas vezes, são entendidos como sinônimos (Pacheco, Alvarenga, Reppold, Piccinini, \& Hutz, 2005). Rutter et al. (1998) definem os comportamentos antissociais como as manifestações que violam as normas sociais. Indivíduos com comportamentos antissociais apresentam comportamentos externalizantes, tais como agressividade, desobediência, atitude desafiante e opositora, dificuldade de controle dos impulsos, roubos, fugas, entre outros. Quando intensos e persistentes estes comportamentos podem evoluir, ao longo do desenvolvimento, para o Transtorno Desafiador Opositivo (TDO), para o Transtorno de Conduta (TC) ou ainda, em adultos, para o Transtorno de Personalidade Antissocial (Borsa, Hauck \& Pacheco, 2013).

Os comportamentos externalizantes apresentam alta prevalência em crianças brasileiras (Fleitlich-Bilyk \& Goodman, 2004) e de outros contextos culturais (Samarakkody, Fernando, McClure, Perera, \& Silva, 2012). Especificamente, o comportamento agressivo é um tipo de comportamento externalizante que tem sido amplamente discutido na Psicologia e que pode ser descrito como um ato ou conduta que visa causar algum dano físico ou psicológico a alguém ou a um grupo de pessoas (Borsa, 2012; Borsa \& Nunes, 2011; Borsa, Souza, \& Bandeira, 2011). Achenbach (1991) descreve outro tipo de problema de comportamento externalizante comum em crianças e adolescentes, denominado comportamento de quebra de regras. Este é caracterizado por condutas socialmente indesejáveis que violam ou se opõem às normas e regras sociais. São exemplos destes comportamentos o vandalismo, o abuso de álcool e drogas, furtos e mentiras.

Os comportamentos agressivos e de quebra de regras guardam certas semelhanças entre si que justificam analisá-los em uma mesma categoria: se manifestam a partir da relação com o meio, envolvem comportamentos socialmente indesejáveis e quando intensos e persistentes, tendem a ter repercussões negativas ao longo do desenvolvimento (Achenbach, 1991).

O relacionamento entre pares, o processo de socialização e a internalização de regras sociais exigem das crianças e adolescentes o uso de uma série de estratégias de enfrentamento, seja para tolerar frustrações, adiar gratificações ou para lidar com situações de conflito interpessoal (Boeckerts, 1996). Um conceito fundamental para compreender os comportamentos de crianças e adolescentes frente às demandas pessoais e contextuais é o coping. Entende-se por coping os esforços cognitivos ou comportamentais que gerenciam demandas internas e externas, percebidas como uma sobrecarga aos recursos pessoais (Lazarus \& Folkman, 1984). O coping envolve a avaliação que o indivíduo faz de um problema ou evento estressor. Considera, também, as características individuais e do contexto que podem dificultar ou favorecer o enfrentamento deste problema ou evento estressor (Diniz \& Zanini, 2010).

Lochman (1987), Barros e Silva (2006) descrevem que crianças e adolescentes com comportamentos agressivos apresentam uma distorção perceptiva das 
suas relações interpessoais. Tendem a se frustrar com mais facilidade e a perceber maior hostilidade nas ações dos seus pares e nos acontecimentos em geral, mesmo em situações ambíguas em que não há claramente uma intenção provocativa ou agressiva. Além disso, tendem a apresentar baixa habilidade no enfrentamento de problemas, ou seja, um repertório de coping empobrecido. Lochman e Wells (2003) descrevem como um programa de treinamento em habilidades de coping e manejo do estresse com crianças, seus familiares e professores diminuiu o índice de agressões na escola e na sala de aula. Neste estudo, os adolescentes agressivos foram randomicamente distribuídos em três grupos: um primeiro grupo em que somente o adolescente recebeu o programa de treinamento em coping; um segundo grupo em que o adolescente e os adultos a ele relacionados receberam o programa de treinamento; e o terceiro grupo (grupo controle) em que nenhum treinamento foi oferecido. Ao final de um ano os grupos experimentais apresentaram menores taxas de comportamento delinquente e abuso de substâncias quando comparados com o grupo controle. Essa diminuição dos comportamentos foi ainda maior no grupo em que tanto o adolescente como os adultos a ele relacionados receberam o treinamento de coping. Os resultados deste estudo demonstraram não só a eficácia do programa de treinamento mas também evidências de que os comportamentos agressivos podem ser fruto da inabilidade do adolescente em fazer frente a seus problemas. O presente estudo tem como objetivo comparar as estratégias de coping de crianças e adolescentes com altas e baixas pontuações em problemas de comportamento externalizantes e compreender de que maneira esses jovens avaliam seus problemas.

\section{MÉTODO}

\section{Participantes}

Participaram deste estudo um total de 512 crianças e adolescentes, estudantes de escolas públicas $(n=2)$ e privadas $(n=3)$ da cidade de Goiânia. Destes, 410 cursavam o ensino médio e 102 cursavam o ensino fundamental no momento da coleta de dados. Do total dos 410 participantes que cursavam o ensino médio, 232 eram do sexo feminino (56,6\%), e 171 do sexo masculino (41,7\%). Sete participantes não informaram o sexo. A idade para esta sub-amostra variou de 16 a 18 anos incompletos $(M=17,24 ; D P=0,66)$. Dos 102 alunos dos ensinos fundamental, 61(59\%) eram do sexo masculino e 41 (41\%) do sexo feminino. A idade desta sub-amostra variou de 11 a 15 anos completos $(M=12,80 ; D P=0,98)$. A seleção dos participantes obedeceu o critério de conveniência e a todos foi solicitada a autorização dos pais mediante o Termo de Consentimento Livre e Esclarecido (TCLE). Somente aqueles que devolveram o TCLE assinado participaram do estudo, o que justificou a variação no número de participantes segundo o grupo etário.

\section{Instrumentos}

Para investigar a avaliação dos problemas bem como as estratégias de coping das crianças e adolescentes, foi utilizado o Coping Response Inventory- $Y$ (CRI-Y). O CRI-Y, desenvolvido por Moos (1993), visa medir os esforços cognitivos e comportamentais dos indivíduos no enfrentamento a problemas e divide-se em três partes: a) descrição da situação mais estressante ou difícil vivenciada nos últimos 12 meses pelo participante. Esta primeira é composta de uma única questão aberta onde a criança ou adolescente é convidado a escrever qual o problema mais estressante que vivenciou nos últimos 12 meses; b) dez itens referentes à apreciação do problema descrito previamente, que engloba questões relativas, prejuízos causados, lócus de controle, se o problema já está resolvido, entre outras e; c) 48 itens relativos à descrição de comportamentos que caracterizam as formas de enfrentamento de problemas utilizadas pelos participantes. Esses itens são respondidos por meio de uma escala tipo Likert de quatro pontos e as respostas se agrupam, posteriormente, pela soma direta dos itens, em oito escalas de estratégias de coping: análise lógica, reavaliação positiva, busca de apoio social, resolução de problemas, reavaliação cognitiva, aceitação ou resignação, busca de gratificação alternativa e descarga emocional. O primeiro grupo de quatro escalas mede o coping de aproximação ao problema ou enfrentamento direto (análise lógica, reavaliação positiva, busca de orientação e apoio e resolução de problemas); o segundo grupo de quatro escalas (evitação cognitiva, aceitação-resignação, busca de gratificação alternativa e descarga emocional) mede o coping de evitação ao problema ou enfrentamento indireto. As duas primeiras escalas de cada grupo medem estratégias de coping cognitivas; a terceira e quarta escalas de cada grupo medem estratégias de coping comportamental (Moos, 1993).

O CRI-Y (Moos, 1993) foi traduzido e validado para o português por Zanini, Mendonça, Forns e Kirchner (2010) e apresentou índices de consistência interna satisfatórios e semelhantes aos encontrados na versão original. A versão em português apresentou valores de alphas de Cronbach entre 0,45 e 0,67 para meninos e 0,50 e 0,72 para meninas para as escalas 
específicas. Para a escala de coping de aproximação os alfas foram de 0,79 para meninos e meninas e para coping de evitação os alfas foram de 0,71 para meninas 0,76 para meninos (Zanini et al., 2010). Tanto na versão em inglês como na brasileira não foram realizados estudos de normatização e os escores são interpretados a partir das comparações entre médias dos escores brutos (Moos, 1993).

Para avaliar os problemas de comportamento foi utilizado o Youth Self Report (YSR, Achenbach, 1991; 1995), adaptado e validado para o contexto brasileiro por Rocha (2012). Trata-se de um questionário de autorrelato, empiricamente baseado, que visa avaliar os problemas de comportamento e de competência social de adolescentes entre 11 e 18 anos. Esse instrumento é composto por duas partes. A primeira contém sete questões que avaliam as competências sociais dos adolescentes. A segunda é composta por 112 itens que avaliam diferentes tipos de problemas de comportamento internalizantes e externalizantes. Estes itens são divididos nas seguintes escalas: ansiedade/depressão, isolamento/depressão, problemas somáticos, problemas sociais, problemas de pensamento, problemas de atenção, quebra de regras e comportamento agressivo.

O YSR foi construído a partir de dados de pesquisas empíricas desenvolvidas em diversos contextos e culturas (Achenbach, 1991). Os resultados obtidos naqueles estudos foram analisados estatisticamente para identificar os padrões de problemas mais frequentemente pontuados por diferentes avaliadores. Para este estudo, apenas a segunda parte do instrumento foi analisada e, mais especificamente, os problemas externalizantes. Os dados de normatização do instrumento para a amostra norte-americana indicam que escores $\mathrm{t}$ (escores padronizados) até 67 pontos são considerados normais, entre 67 e 70 são considerados borderline e maiores que 70 indicam pontuações clínicas naquela subescala. Contudo, em função do instrumento não contar com dados de normatização para o contexto brasileiro, o presente estudo foi conduzido utilizando as pontuações brutas.

\section{Procedimentos de Coleta de Dados}

Após a aprovação do projeto no Comitê de Ética em Pesquisa da Pontifícia Universidade Católica de Goiás sob o protocolo número 0113.0.168.000-06 e mediante a autorização das escolas para a execução da pesquisa, foram programadas duas sessões para a explicação e a aplicação dos questionários. Estes foram administrados de forma coletiva em sala de aula, aplicados por estudantes e psicólogos previamente orientados e treinados para esta finalidade.
$\mathrm{Na}$ primeira sessão, os alunos receberam esclarecimentos acerca dos objetivos da pesquisa e do sigilo. Oportunamente, abriu-se espaço para esclarecimento de dúvidas a respeito dos instrumentos e da leitura e assinatura do termo de consentimento livre e esclarecido. Na segunda sessão foram aplicados os questionários CRI-Y e YSR. Embora este estudo não tenha tido caráter interventivo, foi oferecido para todos os participantes da pesquisa que apresentaram necessidade e/ou demonstraram interesse pela possibilidade de apoio psicológico, conforme estabelecido pelo Comitê de Ética em Pesquisa vinculado à instituição onde este estudo foi desenvolvido.

\section{Procedimentos de Análise de Dados}

As análises foram realizadas mediante estatísticas descritivas e inferenciais. Para avaliar possíveis diferenças entre sexo nas pontuações obtidas nos questionários CRI-Y (escalas de coping e avaliação de problemas) e YSR (comportamento agressivo e de quebra de regras), realizou-se testes $t$ de Student e Análise de Variância (ANOVA).

\section{RESULTADOS}

No que se refere às escalas comportamentos agressivos e de quebra de regras, não foram observadas diferenças significativas entre as pontuações de meninos e meninas (Tabela 1). Como as médias nas pontuações não apresentaram variação significativa em função do sexo do participante as demais análises foram realizadas tomando-se a amostra total e não segmentando por sexo.

Para comparar a forma de avaliação e enfrentamento do problema segundo as pontuações nas escalas quebra de regra e comportamento agressivo foi realizada uma classificação da amostra com base nos quartis das pontuações destas escalas. Desta forma, a amostra foi subdividida em três grupos segundo as pontuações em percentis: grupo 1 (pontuações abaixo 8,0 para quebrar regras e 13,0 para comportamento agressivo), correspondente aos participantes com menores pontuações; grupo 2 (pontuações entre 18,0 e 21,0 para quebrar regras e 23,0 a 27,0 para comportamento agressivo.), correspondente aos participantes com pontuações médias; e grupo 3, correspondente aos participantes com maiores pontuações nas escalas (pontuações acima de 21,0 para quebrar regras e 27,0 para comportamento agressivo).

Uma análise de variância (ANOVA) foi realizada para avaliar como os participantes com diferentes pontuações (baixa, média e alta) nas escalas quebra 
TABELA 1

Média, desvio padrão, descrição do quartil, mínimo-máximo, e comparação de média segundo técnica de $t$ student entre problemas de comportamento do tipo quebrar regras e comportamento agressivo e sexo.

\begin{tabular}{|c|c|c|c|c|}
\hline & \multicolumn{2}{|c|}{ Quebrar Regras } & \multicolumn{2}{|c|}{ Comportamento agressivo } \\
\hline & Meninos & Meninas & Meninos & Meninas \\
\hline Média (DP) & $15,35(8,41)$ & $15,78(7,13)$ & $20,03(9,19)$ & $21,63(8,73)$ \\
\hline$t$ student & \multicolumn{2}{|c|}{$t=-0,53 p=0,60$} & \multicolumn{2}{|c|}{$t=-1,73 p=0,08$} \\
\hline \multicolumn{5}{|l|}{ Percentil } \\
\hline 25 & \multicolumn{2}{|c|}{8,0} & \multicolumn{2}{|c|}{13,0} \\
\hline 50 & \multicolumn{2}{|c|}{18,0} & \multicolumn{2}{|c|}{23,0} \\
\hline 75 & \multicolumn{2}{|c|}{21,0} & \multicolumn{2}{|c|}{27,0} \\
\hline Mínimo-Máximo & \multicolumn{2}{|c|}{$0-37$} & \multicolumn{2}{|c|}{$2-40$} \\
\hline
\end{tabular}

de regras e comportamento agressivo avaliam e enfrentam seus problemas. Foi conduzido o teste post hoc de Scheffé para identificar os grupos com diferenças significativas nas médias. Indivíduos com média e alta pontuação nesta escala prezam mais por saber sobre seus problemas quando comparados ao grupo com baixa pontuação $(F=13,51, p<0,001)$. Porém são os indivíduos com baixa e média pontuação nesta escala os que relatam ter tido menos tempo para enfrentar seus problemas $(F=8,11, p<0,001)$. Os indivíduos com baixa pontuação na escala quebra de regras apresentaram maiores escores nos itens "se seus problemas já estão resolvidos" $(F=10,99, p<0,001) \mathrm{e}$ "se as coisas estão bem agora" $(F=10,90, p<0,001)$, quando comparados aos indivíduos com média ou alta pontuação (Tabela 2).

Conforme demonstrado na Tabela 3 os participantes com menores pontuações em comportamento agressivo foram aqueles que "avaliaram menos que o problema ia lhes acontecer" $(F=1,11, p<0,001)$, "ter tido tempo para enfrentarem o problema" $(F=3,14, p \leq 0,05)$, e "terem visto o problema como desafio" $(F=12,80, p<0,001)$. Contudo, foram os que avaliaram significativamente mais "terem resolvido seus problemas" $(F=15,23$, $p<0,001)$ e que "as coisas agora iam bem" $(F=9,78$, $p<0,001)$.

TABELA 2

Comparação de média por meio da ANOVA e post hoc de Scheffé entre comportamento de quebrar regras (baixo, médio e alto), e avaliação do problema.

\begin{tabular}{|c|c|c|c|c|c|}
\hline \multirow[t]{2}{*}{ Avaliação do problema* } & \multicolumn{3}{|c|}{$\begin{array}{c}\text { Quebra de regras } \\
\text { Média }(D P)\end{array}$} & \multicolumn{2}{|c|}{ ANOVA } \\
\hline & Baixo & Médio & Alto & $F$ & $p$ \\
\hline Experiência com problema & $0,87(1,11)$ & $0,71(1,19)$ & $0,59(1,12)$ & 1,30 & 0,27 \\
\hline Saber que aconteceria & $1,95(1,35)^{\mathrm{a}}$ & $2,55(0,95)^{\mathrm{b}}$ & $2,69(0,78)^{\mathrm{b}}$ & 13,51 & $<0,001$ \\
\hline Tempo para enfrentar & $1,21(1,34)^{\mathrm{a}}$ & $1,19(1,23)^{\mathrm{a}}$ & $1,87(1,21)^{\mathrm{b}}$ & 8,11 & $<0,001$ \\
\hline Problema como prejuízo & $1,94(1,29)$ & $1,98(1,22)$ & $1,75(1,22)$ & 0,85 & 0,043 \\
\hline Problema como desafio & $1,79(1,38)^{\mathrm{a}}$ & $2,42(1,04)^{b}$ & $2,22(1,14)^{b}$ & 9,75 & $<0,001$ \\
\hline Causado por você & $0,87(1,31)$ & $1,07(1,24)$ & $1,27(1,24)$ & 1,97 & 0,14 \\
\hline Causado por outro & $1,39(1,39)$ & $1,19(1,22)$ & $1,17(1,21)$ & 0,89 & 0,41 \\
\hline Gerou coisas boas & $1,56(1,34)$ & $1,79(1,26)$ & $1,63(1,31)$ & 1,13 & 0,32 \\
\hline Está resolvido & $1,70(1,29)^{\mathrm{a}}$ & $0,97(1,36)^{b}$ & $0,96(1,19)^{\mathrm{b}}$ & 10,99 & $<0,001$ \\
\hline Está tudo bem & $1,79(1,27)^{\mathrm{a}}$ & $1,07(1,23)^{b}$ & $1,22(1,25)^{\mathrm{b}}$ & 10,90 & $<0,001$ \\
\hline
\end{tabular}

* Descrição aproximada das perguntas relativas à avaliação do problema. O autor e editora não autorizam a divulgação total das questões do instrumento. Nota: Diferentes sobre-escritos indicam médias significativamente diferentes. 
TABELA 3

Comparação de média por meio da ANOVA e post hoc de Scheffé entre comportamento agressivo (baixo, médio e alto), e avaliação do problema.

\begin{tabular}{|c|c|c|c|c|c|}
\hline \multirow[t]{2}{*}{ Avaliação do problema* } & \multicolumn{3}{|c|}{$\begin{array}{c}\text { Comportamento Agressivo } \\
\text { Média }(D P)\end{array}$} & \multicolumn{2}{|c|}{$A N O V A$} \\
\hline & Baixo & Médio & Alto & $F$ & $p$ \\
\hline Experiência com problema & $0,87(1,13)$ & $0,67(1,08)$ & $0,66(1,10)$ & 1,20 & 0,30 \\
\hline Saber que aconteceria & $1,95(1,36)^{\mathrm{a}}$ & $2,48(0,98)^{\mathrm{b}}$ & $2,62(0,90)^{b}$ & 11,11 & $<0,001$ \\
\hline Tempo para enfrentar & $1,15(1,32)^{\mathrm{a}}$ & $1,28(1,26)^{\mathrm{a}}$ & $1,59(1,22)^{b}$ & 3,14 & $<0,05$ \\
\hline Problema como prejuízo & $1,88(1,33)$ & $1,92(1,23)$ & $1,87(1,24)$ & 0,47 & 0,95 \\
\hline Problema como desafio & $1,74(1,39)^{\mathrm{a}}$ & $2,40(1,02)^{b}$ & $2,42(1,01)^{b}$ & 12,80 & $<0,001$ \\
\hline Causado por você & $0,90(1,30)$ & $0,95(1,22)$ & $1,22(1,21)$ & 1,89 & 0,15 \\
\hline Causado por outro & $1,37(1,37)$ & $1,23(1,23)$ & $1,21(1,23)$ & 0,52 & 0,60 \\
\hline Gerou coisas boas & $1,67(1,36)$ & $1,70(1,28)$ & $1,86(1,22)$ & 0,68 & 0,51 \\
\hline Está resolvido & $1,78(1,28)^{\mathrm{a}}$ & $1,11(1,40)^{\mathrm{b}}$ & $0,76(1,11)^{b}$ & 15,23 & $<0,001$ \\
\hline Está tudo bem & $1,76(1,30)^{\mathrm{a}}$ & $1,20(1,24)^{\mathrm{b}}$ & $1,01(1,22)^{\mathrm{b}}$ & 9,78 & $<0,001$ \\
\hline
\end{tabular}

* Descrição aproximada das perguntas relativas à avaliação do problema. O autor e editora não autorizam a divulgação total das questões do instrumento. Nota: Diferentes sobre-escritos indicam médias significativamente diferentes.

De uma forma geral, a apreciação do problema de grupos baixos, médios e altos em quebra de regras e comportamento agressivo apresentaram um perfil muito semelhante. Tomados em conjunto, os dados indicaram que os grupos de crianças e adolescentes com pontuações baixa, média ou alta em quebra de regras ou comportamento agressivo tendem a apresentar padrões comuns na apreciação de seus problemas.

De acordo com a Tabela 4, observou-se que crianças e adolescentes com diferentes pontuações na escala de quebra de regras enfrentam seus problemas de forma distinta. Aqueles com pontuações mais baixas tenderam a utilizar menos estratégias do tipo reavaliação positiva do problema $(F=18,95, p<0,001)$, busca de apoio $(F=7,29, p<0,001)$, e resolução do problema $(F=7,94$, $p<0,001)$, o que configura um uso significativamente menor das estratégias de aproximação quando comparados aos seus companheiros com pontuações médias ou altas na escala de quebra de regras $(F=13,33$, $p<0,001)$.

TABELA 4

Comparação de média por meio da ANOVA e post hoc de Scheffé entre quebrar regras (baixo, médio e alto), e enfrentamento ao problema.

\begin{tabular}{|c|c|c|c|c|c|}
\hline \multirow[t]{2}{*}{ Estratégia de enfrentamento } & \multicolumn{3}{|c|}{$\begin{array}{c}\text { Quebra de Regras } \\
\text { Média (DP) }\end{array}$} & \multicolumn{2}{|c|}{ ANOVA } \\
\hline & Baixo & Médio & Alto & $F$ & $p$ \\
\hline Análise lógica & $10,00(11,24)$ & $11,25(3,25)$ & $11,42(2,98)$ & 1,49 & 0,23 \\
\hline Reavaliação positiva & $8,37(4,09)^{\mathrm{a}}$ & $11,24(3,76)^{\mathrm{b}}$ & $11,04(3,81)^{b}$ & 18,95 & $<0,001$ \\
\hline Busca de apoio & $6,63(4,13)^{\mathrm{a}}$ & $8,43(4,63)^{b}$ & $9,00(4,03)^{b}$ & 7,29 & $<0,001$ \\
\hline Resolução de problema & $8,57(4,85)^{\mathrm{a}}$ & $10,55(3,83)^{\mathrm{b}}$ & $10,10(3,11)^{b}$ & 7,94 & $<0,001$ \\
\hline Evitação cognitiva & $8,31(4,03)$ & $8,41(4,06)$ & $9,10(3,94)$ & 0,92 & 0,40 \\
\hline Aceitação resignação & $7,42(4,36)$ & $6,87(3,97)$ & $7,64(3,51)$ & 1,21 & 0,30 \\
\hline Busca de gratificação & $8,06(4,66)^{\mathrm{a}}$ & $8,76(3,59)$ & $9,82(3,78)^{\mathrm{b}}$ & 3,96 & $<0,05$ \\
\hline Descarga emocional & $6,26(3,97)$ & $7,88(3,70)$ & $8,93(3,68)$ & 10,75 & $<0,001$ \\
\hline Aproximação & $33,63(16,31)^{\mathrm{a}}$ & $41,54(11,47)^{b}$ & $41,72(10,36)^{b}$ & 13,33 & $<0,001$ \\
\hline Evitação & $30,15(11,23)^{\mathrm{a}}$ & $31,97(10,05)$ & $35,28(9,46)^{b}$ & 4,80 & $<0,001$ \\
\hline
\end{tabular}

Nota: Diferentes sobre-escritos indicam médias significativamente diferentes. 
Além disso, crianças e adolescentes com baixas pontuações na escala de quebra de regras também relataram enfrentar seus problemas utilizando menos estratégias de busca de gratificação alternativa $(F=8,06, p \leq 0,05)$ e descarga emocional $(F=10,75$, $p<0,001)$, configurando um uso mais baixo das estratégias de evitação do problema $(F=4,80, p \leq 0,05)$ quando comparado aos seus companheiros com pontuações mais altas nesta escala.

Segundo demonstrado na Tabela 5, o uso de estratégias de enfrentamento para crianças e adolescentes com diferentes pontuações em comportamento agressivo apresentou algumas características semelhantes às do grupo de crianças e adolescentes com problemas do tipo quebra de regras. Assim como no primeiro grupo analisado, crianças e adolescentes com baixas pontuações em comportamento agressivo tenderam a enfrentar seus problemas utilizando menos estratégias do tipo reavaliação positiva $(F=17,37$, $p<0,001)$, busca de apoio ( $\mathrm{F}=5,85, p=0,00)$ e resolução de problemas $(F=7,16, p<0,001)$, perfazendo um menor uso das estratégias do tipo aproximação do problema $(F=11,50, p<0,001)$.

A estratégia de coping aceitação e resignação apresentou um uso diferencial segundo o grupo analisado: crianças e adolescentes com baixos índices de comportamento agressivo utilizaram pouco esta estratégia, embora significativamente mais que seus companheiros com pontuações médias e significativamente menos que seus companheiros com altas pontuações nesta escala $(F=8,50, p<0,001)$. Além disso, este grupo não apresentou diferença significativa no uso da estratégia de busca de gratificação alternativa $(F=1,82, p=0,16)$. No entanto, assim como no outro grupo, são as crianças e adolescentes com menores pontuações na escala de comportamento agressivo que utilizaram significativamente menos as estratégias do tipo descarga emocional $(F=11,62, p<0,001)$, perfazendo um menor uso de estratégias de evitação para os grupos com pontuações baixas e médias na escala de comportamento agressivo $(F=7,64, p<0,001)$ (ver Tabela 5).

\section{DISCUSSÃO}

Embora diferentes estudos apontem que meninos apresentam significativamente mais problemas de agressividade e de quebra de regras que as meninas tanto no Brasil (Emerich, et al., 2012), como em outras culturas (Achenbach, 1991, 1995; Ivanova et al., 2007; Lochman, 1987), neste estudo não foram encontradas diferenças significativas entre ambos os grupos. Além disso, não foram observadas pontuações muito altas nas escalas de problemas de comportamento como quebra de regras ou comportamentos agressivos sugerindo que, neste estudo, meninos e meninas apresentam boa capacidade de adaptação no que se refere a estes problemas de comportamento.

O presente estudo corrobora os achados de Lochman (1987) e Barros e Silva (2006) sobre a existência de uma distorção perceptiva e uma menor habilidade de coping entre crianças e adolescentes que apresentam comportamentos agressivos. Nesta amostra, crianças e adolescentes com maiores pontuações tanto nas escalas

TABELA 5

Comparação de média por meio da ANOVA e post hoc de Scheffé entre comportamento agressivo (baixo, médio e alto), e enfrentamento ao problema.

\begin{tabular}{|c|c|c|c|c|c|}
\hline \multirow[t]{2}{*}{ Estratégia de enfrentamento } & \multicolumn{3}{|c|}{$\begin{array}{c}\text { Comportamento agressivo } \\
\text { Média }(D P)\end{array}$} & \multicolumn{2}{|c|}{ ANOVA } \\
\hline & Baixo & Médio & Alto & $F$ & $p$ \\
\hline Análise lógica & $9,95(11,09)$ & $11,09(3,07)$ & $11,80(3,29)$ & 2,18 & 0,11 \\
\hline Reavaliação positiva & $8,64(4,06)^{\mathrm{a}}$ & $11,12(3,76)^{\mathrm{b}}$ & $11,45(3,61)^{b}$ & 17,37 & $<0,001$ \\
\hline Busca de apoio & $6,83(4,29)^{\mathrm{a}}$ & $7,95(4,52)^{b}$ & $9,02(4,44)^{b}$ & 5,85 & $<0,001$ \\
\hline Resolução de problema & $8,63(4,79)^{\mathrm{a}}$ & $10,52(3,86)^{b}$ & $9,91(3,25)^{\mathrm{b}}$ & 7,16 & $<0,001$ \\
\hline Evitação cognitiva & $8,20(4,22)$ & $8,26(4,14)$ & $9,19(3,91)$ & 1,90 & 0,15 \\
\hline Aceitação resignação & $7,70(4,57)^{\mathrm{a}}$ & $6,36(3,79)^{b}$ & $8,28(3,55)^{\mathrm{a}}$ & 8,50 & $<0,001$ \\
\hline Busca de gratificação & $8,28(4,69)$ & $8,79(3,73)$ & $9,38(3,61)$ & 1,82 & 0,16 \\
\hline Descarga emocional & $6,33(3,95)$ & $7,59(3,62)$ & $8,94(3,73)$ & 11,62 & $<0,001$ \\
\hline Aproximação & $34,16(16,14)^{a}$ & $40,80(11,38)^{b}$ & $42,19(10,68)^{b}$ & 11,50 & $<0,001$ \\
\hline Evitação & $30,61(11,61)^{\mathrm{a}}$ & $30,89(9,50)^{\mathrm{a}}$ & $35,66(10,09)^{\mathrm{b}}$ & 7,64 & $<0,001$ \\
\hline
\end{tabular}

Nota: Diferentes sobre-escritos indicam médias significativamente diferentes. 
de quebra de regras como na escala de comportamento agressivo avaliaram seus problemas de forma semelhante e significativamente diferente dos demais grupos com pontuações mais baixas. De acordo com os dados, este grupo de crianças e adolescentes tiveram mais ciência de que o problema vai lhes acontecer, relataram ter mais tempo para enfrentá-lo e sentiram o problema como um desafio. No entanto, ainda assim, avaliaram resolver menos seus problemas ou que as coisas estão bem após seu enfrentamento. Este mesmo grupo usou mais reavaliação positiva, mais busca de apoio, mais resolução de problemas e descarga emocional.

Crianças e adolescentes com altas pontuações em ambas as escalas utilizam significativamente mais estratégias de coping (tanto de aproximação quanto de evitação) para o enfrentamento de seus problemas. Desta forma, ainda utilizando de um repertório comportamental e cognitivo significativamente maior que seus companheiros, tendo mais tempo para este enfrentamento e sabendo que teriam que enfrentar este problema, as crianças e adolescentes que apresentam mais comportamentos agressivos relataram não conseguirem resolver seus problemas.

Aldwin (1994) já havia apontado a diferença entre quantidade e qualidade das estratégias de coping utilizadas por adolescentes. A autora aponta que o maior uso de estratégias de coping nem sempre está relacionado a uma melhor resolução do problema e que embora as meninas utilizem mais estratégias de coping (tanto as focadas no problema como as focadas na emoção) são elas as que apresentam maiores problemas de comportamento quando comparadas aos meninos. A autora discute os dados com base na eficácia da estratégia de coping utilizada, evidenciando a importância da assertividade na escolha e avaliação da eficácia da estratégia.

Embora tenham apresentado um conjunto significativamente maior de estratégias de coping, as crianças e adolescentes do presente estudo com altas pontuações em quebra de regras e comportamento agressivo não reportaram utilizar aquelas estratégias relacionadas a uma maior eficácia, como é o caso da estratégia de resolução do problema. Estudos anteriores demonstram que esta estratégia é a que mais se relaciona com menores pontuações em psicopatologia ou problemas de comportamento (Zanini et al., 2010) e, portanto, poderia ser a que melhor indicaria saúde mental ou boa adaptação social daqueles que utilizam. Ao contrário, este grupo de crianças e adolescentes tenderam a utilizar significativamente mais o conjunto de estratégias associadas a uma maior desadaptação social, sobretudo a estratégia do tipo descarga emo- cional cujos índices de correlação com problemas de comportamento são sempre superiores a qualquer outra estratégia de coping (Zanini et al., 2010).

Por fim, o presente estudo demonstrou que, no que tange a influência no uso das estratégias de coping, a manifestação de comportamentos agressivos ou de quebra de regras apresentaram características muito semelhantes. Apenas para a estratégia do tipo "busca de gratificação alternativa" foi encontrada diferença. Tal diferença no uso desta estratégia entre os grupos de comportamento agressivo e quebra de regras justifica-se, pois esta estratégia avalia os mesmos comportamentos utilizados como critério para identificação de comportamentos de quebra de regras tais como fumar, beber, ou fazer coisas que dão prazer mas que, de alguma forma, podem não ser consideradas adequadas, ou que o adolescente ou criança não faria em situações de não estresse (Moos, 1993). De fato, Moos (1993) já havia descrito relações significativas entre esta estratégia e problemas de comportamento como uso de álcool e drogas. Neste sentido, um uso aumentado desta forma de enfrentamento pode ser indicativo de maior risco social e da necessidade de uma avaliação mais cuidadosa.

\section{CONSIDERAÇÕES FINAIS}

O presente estudo teve como objetivo comparar como crianças e adolescentes com altas médias e baixas pontuações em problemas de comportamento externalizantes (do tipo agressivo e quebra de regras) avaliam e enfrentam seus problemas. Em conjunto, os dados apresentados neste estudo parecem corroborar os postulados de Lochman (1987) e Lochman e Wells (2003) que apontam a existência de uma distorção perceptiva e baixas habilidades de coping entre crianças e adolescentes com problemas de comportamento agressivo (e, neste estudo, de quebra de regras também). Neste sentido, pode-se pressupor que, assim como realizado por Lochman e Wells (2003) o desenvolvimento de um programa de manejo de estresse, que inclua um trabalho cognitivo para correção das distorções cognitivas, e um treinamento em habilidades de enfrentamento a problemas em crianças e adolescentes poderia, prevenir comportamentos agressivos e de quebra de regras. Desta forma, sugere-se que estudos posteriores desenvolvam e avaliem a eficácia de programas de prevenção e dos comportamentos agressivos por meio do trabalho das habilidades de coping.

Os dados apresentados aqui, porém, devem ser tomados com cautela, pois trata-se de um estudo de corte transversal, com população comunitária e com 
medidas de autorrelato. Sendo assim, apresenta as fragilidades metodológicas deste tipo de desenho. Primeiramente, as medidas são relacionais e não oferecem possibilidades de investigação de causalidade. Tanto o fato de avaliar e enfrentar os problemas de forma diferente pode estar levando ao desenvolvimento de comportamento agressivo e de quebra de regra como vice versa. Além disso, utilizou-se como parâmetro a distribuição das pontuações nas escalas de quebra de regra e comportamento agressivo para classificação dos grupos analisados. Esses grupos, entretanto, não refletem as diferenças comportamentais que poderiam ser encontradas entre grupos de crianças e adolescentes sem problemas de comportamento agressivo e de quebra de regras e com problemas em nível clínico. Neste sentido, estudos posteriores poderiam avaliar as diferenças na avaliação e coping entre população clínica e comunitária. Além disso, em função do espaço e da discussão que seria necessária, deixou-se para outro momento a avaliação do tipo de problema vivenciado pelos participantes da pesquisa e a influência da idade na avaliação e seleção das estratégias de coping. Estudos posteriores poderiam esclarecer a relação entre o tipo de problema vivenciado, o uso de estratégias de coping e sua relação com comportamentos agressivos assim como a influência da idade em cada uma dessas variáveis ou no conjunto de sua relação.

Por fim, o presente estudo utilizou-se de medidas de autorrelato e que, portanto, estão sujeitas a interferências relacionadas ao estilo de resposta do sujeito, sua percepção e desejabilidade social. Contudo, apesar destas limitações, o presente estudo oferece uma descrição das diferenças encontradas nas avaliações e enfrentamento de problemas de crianças e adolescentes que apresentam níveis diferentes de comportamentos externalizantes o que poderia orientar ações que visem prevenir estes comportamentos.

\section{REFERÊNCIAS}

American Psychiatric Association (APA). (2003). DSM-IV-TR - Manual diagnóstico e estatístico de transtornos mentais (4⿳亠丷厂 ed.). Porto Alegre: Artmed.

Achenbach, T. M. (1991). Manual for the Youth Self-Report and 1991 Profile. Burlington, VT: University of Vermont Department of Psychiatry.

Achenbach, T. M. (1995). Developmental Issues in Assessment, Taxonomy and Diagnosis of child and adolescent psychopathology. In D. Cichetti \& D. J. Cohen (Eds.). Developmental Psychopathology (pp. 57-80). New York: Wiley and Sons.

Achenbach, T. M. \& Rescorla, L. A. (2010). Mental health pratitioners'guide for the Achenbach System of Empirically Based Assessment (ASEBA) (7th ed.). Burlington,Vermont, Estados Unidos: University of Vermont.

Aldwin, C. (1994). Stress, Coping and Development: an integrative perspective. New York London: The Guilford Press.

Anselmi, L., Fleitlich-Bilyk, B., Menezes, A. M. B., Araújo, C. L., \& Rohde, L. A. (2010). Prevalence of psychiatric disorders in a Brazilian birth cohort of 11-year-olds. Social Psychiatry and Psychiatric Epidemiology, 45(1), 135-142. http://dx.doi.org/10.1007/s00127-009-0052-2

Barros, P. \& Silva, F. B. N. (2006). Origem e manutenção do comportamento agressivo na infância e adolescência. Revista Brasileira de Terapias Cognitivas 2(1), 55-66.

Bolsoni-Silva, A. T. \& Del Prette, A. (2003). Problemas de comportamento: um panorama da área. Revista Brasileira de Terapia Comportamental e Cognitiva, 5(2), 91-103.

Bordin, I. A., Rocha, M. M., Paula, C. S., Teixeira, M. C., Achenbach, T. M., Rescorla, L. A., Silvares, E. F. M. (2013). Child Behavior Checklist (CBCL), Youth Self-Report (YSR) and Teacher's Report Form (TRF): an overview of the development of the original and Brazilian versions. Cad. Saúde Pública, 29(1),13-28. http://dx.doi.org/10.1590/ S0102-311X2013000100004

Borsa, J. C. (2012). Adaptação e validação transcultural do Questionário de Comportamentos Agressivos e Reativos entre Pares (Q-CARP). Tese de Doutorado não-publicada. Universidade Federal do Rio Grande do Sul, Porto Alegre.

Borsa, J. C., Pacheco, J. T. B., Hauck Filho, N. (2013). Transtorno de Personalidade Antissocial: Etiologia e Fatores de Risco. In Lucas de Francisco Carvalho e Ricardo Primi (Org.). Perspectivas em psicologia dos transtornos da personalidade: implicações teóricas e práticas (pp. 498-519). São Paulo: Casa do Psicólogo.

Borsa, J. C., Souza, D. S., \& Bandeira, D. R. (2011). Prevalência dos problemas de comportamento em uma amostra de crianças do Rio Grande do Sul. Psicologia: Teoria e Prática, 13(2), 15-29.

Borsa, J. C. \& Nunes, M. L. T. (2011). Prevalência de problemas de comportamento em uma amostra de crianças escolares da cidade de Porto Alegre. Aletheia, 34(1), 32-46.

Diniz, S. S. \& Zanini, D. S. (2010). Relação entre fatores de personalidade e estratégias de coping em adolescentes. Psico-USF, 15(1), 71-80. http://dx.doi.org/10.1590/S1413-82712010000100008 
Emerich, D. R., Rocha, M. M., \& Silvares, E. F. M. (2012). Diferenças quanto ao gênero entre escolares brasileiros avaliados pelo inventário de comportamentos para crianças e adolescentes (CBCL/6-18). Psico, 43(3), 380-387.

Fleitlich-Bilyk, B. \& Goodman, R. (2004). Prevalence of child and adolescent psychiatric disorders in Southeast Brazil. Journal of the American Academy of Child and Adolescent Psychiatry, 43(6), 727-734. http://dx.doi.org/10.1097/01. chi.0000120021.14101.ca

Homem, T. C., Gaspar, M. S., Azevedo, A. F., \& Canavarro, M. C. (2013). Perturbações de comportamento externalizante em idade pré-escolar: O caso específico da perturbação de oposição. Análise Psicológica, 31(1), 31-48. http://dx.doi. org/10.14417/ap.658

Ivanova, M., Achenbach, T. M., Dumenci, L., Bilenberg, N., Broberg, A. G., Döpfner, M., et al. (2007). The generalizability of the Youth Self-Report Syndrome Structure in 23 Societies. Journal of Consulting and Clinical Psychology, 75(5), 729-738. http://dx.doi.org/10.1037/0022-006X.75.5.729

Lazarus, R. S. \& Folkman, S. (1984). Stress, Appraisal, and Coping. New York: Springer.

Lochman, J. E. (1987). Self and peer perceptions and attributional biases of aggressive and nonaggressive boys in dyadic interactions. Journal of Consulting and Clinical Psychology, 55(3), 404-410. http://dx.doi.org/10.1037/0022006X.55.3.404

Lochman, J. E. \& Wells, K. C. (2003). Effectiveness of the coping power program and of classroom intervention with aggressive children: outcomes at a 1-year follow-up. Behavior Therapy, 34(4), 493-515. http://dx.doi.org/10.1016/ S0005-7894(03)80032-1

Moos, R. H. (1993). Coping Response Inventory Youth Form - Professional Manual. Odessa, Florida: PAR Psychological Assessment Resources, Inc.

Pacheco, J., Alvarenga, P., Reppold, C., Piccinini, C. A., \& Hutz, C. S. (2005). Estabilidade do comportamento anti-social na transição da infância para a adolescência: uma perspectiva desenvolvimentista. Psicologia: Reflexão e Crítica, 18(1), 55-61. http://dx.doi.org/10.1590/S0102-79722005000100008

Rocha, M. M. (2012). Um estudo comparativo entre duas traduções brasileiras do Inventário de Auto-Avaliação para Jovens (YSR). Tese de Doutorado não publicada. Universidade de São Paulo, São Paulo.

Rutter, M., Giller, H., \& Hagell, A. (1998). Antisocial behavior by young people. Cambridge: Cambridge University Press.

Samarakkody, D., Fernando, D., McClure, R., Perera, H., \& De Silva, H. (2012). Prevalence of externalizing behavior problems in Sri Lankan preschool children: birth, childhood, and sociodemographic risk factors. Social psychiatry and psychiatric epidemiology, 47(5), 757-762. http://dx.doi.org/10.1007/s00127-011-0377-5

Zanini, D. S., Mendonça, H., Forns, M., \& Kirchner, T. (2010). Psychometric properties of Coping Response Inventory with Brazilian adolescents. Psychological Reports, 107(2), 617-628. http://dx.doi.org/10.2466/08.17.20. PR0.107.5.617-628

Autores:

DANIEla SACRAmento ZANINI - Doutora, Professora Adjunta do Departamento de Psicologia e Programa de Pós-graduação em Psicologia da PUC-Goiás.

Juliane CAllegaro Borsa - Doutora, Professora Assistente do Departamento de Psicologia e do Programa de Pós-Graduação em Psicologia da PUC-Rio.

Endereço para correspondência:

Daniela S. Zanini

Rua 9, 769, ap. 702 - Setor Oeste

CEP 74110-100 Goiania, GO, Brasil

Recebido em: 07.08.14

Aceito em: 02.03.15 